\title{
YES1 Drives Lung Cancer Growth and Progression and Predicts Sensitivity to Dasatinib
}

\author{
Irati Garmendia ${ }^{1,2}$, María J. Pajares ${ }^{1,2,3,4}$, Francisco Hermida-Prado ${ }^{3,5}$, Daniel Ajona ${ }^{1,3,4,6}$, Cristina Bértolo ${ }^{1,3}$,
} Cristina Sainz ${ }^{1}$, Amaya Lavín ${ }^{1}$, Ana B. Remírez ${ }^{1}$, Karmele Valenciaa,3,6, Haritz Moreno ${ }^{1}$, Irene Ferrer ${ }^{3,7}$, Carmen Behrens 8,9 , Myriam Cuadrado 3,10, Luis Paz-Ares $3,7,11,12$, Xosé R. Bustelo ${ }^{3,10}$, Ignacio Gil-Bazo ${ }^{3,4,13}$, Daniel Alameda ${ }^{1}$, Fernando Lecanda ${ }^{1,2,3,4}$, Alfonso Calvo ${ }^{1,2,3,4}$, Enriqueta Felip ${ }^{14}$, Montse Sánchez-Céspedes ${ }^{15}$, Ignacio I. Wistuba ${ }^{8,9}$, Rocio Granda-Diaz ${ }^{3,5}$, Juan Pablo Rodrigo ${ }^{3,5}$, Juana María García-Pedrero ${ }^{3,5}$, Ruben Pio ${ }^{1,3,4,6}$, Luis M. Montuenga ${ }^{1,2,3,4 \star}$, and Jackeline Agorreta ${ }^{1,2,3,4 *}$

${ }^{1}$ Program in Solid Tumors, Center for Applied Medical Research, Pamplona, Spain; ${ }^{2}$ Department of Pathology, Anatomy, and Physiology, School of Medicine and ${ }^{6}$ Department of Biochemistry and Genetics, School of Sciences, University of Navarra, Pamplona, Spain; ${ }^{3}$ Centro de Investigación Biomédica en Red de Cáncer, Madrid, Spain; ${ }^{4}$ Navarra Health Research Institute, Pamplona, Spain; ${ }^{5}$ Hospital Universitario Central de Asturias, Instituto de Investigación Sanitaria del Principado de Asturias, Instituto Universitario de Oncología del Principado de Asturias (IUOPA), Universidad de Oviedo, Oviedo, Spain; ${ }^{7}$ Lung Cancer Clinical Research Unit and Centro Nacional de Investigaciones Oncológicas, Madrid, Spain; ${ }^{8}$ Department of Translational Molecular Pathology and ${ }^{9}$ Department of Thoracic/Head and Neck Medical Oncology, The University of Texas MD Anderson Cancer Center, Houston, Texas; ${ }^{10}$ Centro de Investigación del Cáncer, Consejo Superior de Investigaciones Científicas, University of Salamanca, Salamanca, Spain; ${ }^{11}$ Medical Oncology Department, Hospital Universitario Doce de Octubre, Madrid, Spain; ${ }^{12}$ Medical School, Universidad Complutense, Madrid, Spain; ${ }^{13}$ Medical Oncology Department, Clínica Universidad de Navarra, Pamplona, Spain; ${ }^{14}$ Oncology Department, Vall d'Hebron University Hospital and Vall d'Hebron Institute of Oncology, Barcelona, Spain; and ${ }^{15}$ Cancer Epigenetics and Biology Program, Genes and Cancer Group, Bellvitge Biomedical Research Institute, Hospitalet de Llobregat, Barcelona, Spain

ORCID IDs: 0000-0001-7124-6137 (I.G.); 0000-0003-3149-8959 (M.J.P.); 0000-0002-8739-1387 (L.M.M.).

\section{Abstract}

Rationale: The characterization of new genetic alterations is essential to assign effective personalized therapies in non-small cell lung cancer (NSCLC). Furthermore, finding stratification biomarkers is essential for successful personalized therapies. Molecular alterations of YES1, a member of the SRC (proto-oncogene tyrosine-protein kinase Src) family kinases (SFKs), can be found in a significant subset of patients with lung cancer.

Objectives: To evaluate YES1 (v-YES-1 Yamaguchi sarcoma viral oncogene homolog 1) genetic alteration as a therapeutic target and predictive biomarker of response to dasatinib in NSCLC.

Methods: Functional significance was evaluated by in vivo models of NSCLC and metastasis and patient-derived xenografts. The efficacy of pharmacological and genetic (CRISPR [clustered regularly interspaced short palindromic repeats]/Cas9 [CRISPR-associated protein 9]) YES1 abrogation was also evaluated. In vitro functional assays for signaling, survival, and invasion were also performed. The association between YES1 alterations and prognosis was evaluated in clinical samples.
Measurements and Main Results: We demonstrated that YES1 is essential for NSCLC carcinogenesis. Furthermore, YES1 overexpression induced metastatic spread in preclinical in vivo models. YES1 genetic depletion by CRISPR/Cas9 technology significantly reduced tumor growth and metastasis. YES1 effects were mainly driven by mTOR (mammalian target of rapamycin) signaling. Interestingly, cell lines and patient-derived xenograft models with YES1 gene amplifications presented a high sensitivity to dasatinib, an SFK inhibitor, pointing out YES1 status as a stratification biomarker for dasatinib response. Moreover, high YES1 protein expression was an independent predictor for poor prognosis in patients with lung cancer.

Conclusions: YES1 is a promising therapeutic target in lung cancer. Our results provide support for the clinical evaluation of dasatinib treatment in a selected subset of patients using YES1 status as predictive biomarker for therapy.

Keywords: dasatinib; YES1; Src family kinases; predictive biomarker; lung cancer

(Received in original form July 19, 2018; accepted in final form June 4, 2019)

*These two authors share senior authorship.

Correspondence and requests for reprints should be addressed to Luis M. Montuenga, Ph.D., Program in Solid Tumors, Center for Applied Medical Research (CIMA), Pio XII Avenue 55, 31008 Pamplona, Spain. E-mail: Imontuenga@unav.es.

This article has an online supplement, which is accessible from this issue's table of contents at www.atsjournals.org.

Am J Respir Crit Care Med Vol 200, Iss 7, pp 888-899, Oct 1, 2019

Copyright $\odot 2019$ by the American Thoracic Society

Originally Published in Press as DOI: 10.1164/rccm.201807-1292OC on June 5, 2019

Internet address: www.atsjournals.org 


\section{At a Glance Commentary}

\section{Scientific Knowledge on the}

Subject: Identifying a subset of patients with specific druggable mutations is critical to developing personalized treatments that will benefit these specific patients over receiving current state-of-the-art nontargeted therapies. In this context, we identify YES1 (v-YES-1 Yamaguchi sarcoma viral oncogene homolog 1) genomic alteration as a potential stratification biomarker that predicts benefit from SFK (SRC [protooncogene tyrosine-protein kinase $\mathrm{Src}]$ family kinases) inhibitors.

\section{What This Study Adds to the Field:}

In this article, we address both of these challenges. First, we demonstrate that YES1 genetic alterations constitute a potential therapeutic target in both adenocarcinoma and squamous cell carcinoma subtypes. Second, we have shown that YES1 status (amplification and overexpression) is a predictive marker of response to the SFK inhibitor dasatinib in non-small cell lung cancer cells and patient-derived xenografts. This study suggests that YES1 inhibition may represent a novel therapeutic target to control lung cancer dissemination in a selected subset of patients with genetic alterations of this gene.

The treatment landscape for patients with non-small cell lung cancer (NSCLC) with advanced-stage disease has deeply changed over the last years. Next-generation sequencing techniques have allowed the discovery of driver mutations that can be targeted with specific drugs (1). The identification of gain-offunction mutations in EGFR (epidermal growth factor receptor) set the basis for the use of molecular-targeted therapies with EGFR tyrosine kinase inhibitors (2). The discovery of $A L K$ (anaplastic lymphoma kinase) rearrangements accelerated approval of specific inhibitors that led to dramatic improvements in the outcome of selected subgroups of patients (1). However, although lung cancer is the paradigm of genomics-driven oncology, there is still a high proportion of patients with NSCLC (around 50\%) who cannot benefit from targeted therapies (3). Therefore, the identification of new genetic alterations may expand the population who could benefit from these therapeutic strategies.

Previously, we showed the potential clinical relevance of gene amplification of YES1 (v-YES-1 Yamaguchi sarcoma viral oncogene homolog 1) as part of a prognostic signature for patients with stage I or II lung adenocarcinoma (ADC) on the basis of copy number $(\mathrm{CN})$ alterations and clinical profiles (4). YES1 is a nonreceptor cytoplasmic tyrosine kinase that belongs to the SRC family kinases (SFKs). This family consists of nine proteins sharing similar structural architecture (5). SRC has been the most studied member of the family (6). Although several SFK inhibitors have been investigated in a variety of tumors, dasatinib is the only one currently approved in clinical practice, indicated for the treatment of patients with leukemia (7). However, SFK inhibitors have not yet demonstrated benefit in the treatment of solid tumors (1).

YES1 is the only member of the SFKs regulated mainly by gene amplification, and a high correlation has been described between gene $\mathrm{CN}$ and mRNA expression in different tumors (8). Furthermore, YES1 gene amplification is a recurrent alteration in lung squamous cell carcinoma (SCC) (8) as well as in other solid tumors (9-11), together with elevated YES1 expression (12-16).

However, its role in lung cancer has not been deeply explored yet. Interestingly, YES1 gene amplification has been recently linked to resistance to EGFR inhibitors in NSCLC cell lines (17) and patients $(18,19)$.

In the present study, we report that YES1 amplification, which correlates with YES1 overexpression, is an oncogenic driver alteration in NSCLC that induces tumor growth and metastatic spreading. Accordingly, high YES1 protein expression is an independent predictor of poor outcome in NSCLC. In addition, we show that YES1 status is a predictive marker of response to the SFK inhibitor dasatinib in NSCLC cells and patient-derived xenograft (PDX) models. Therefore, we propose that genetic alterations of YES1 drive NSCLC progression and define a subset of patients who may potentially benefit from dasatinib treatment.

Some of the results of these studies have been previously reported in the form of abstracts (20-22).

\section{Methods}

See the online supplement for additional details.

\section{Patient Samples}

A series of 116 patients with NSCLC who underwent surgical resection at the Clínica Universidad de Navarra (CIMA-CUN) from 1999 to 2016. A validation series of 222 patients with NSCLC diagnosed from 2003 to 2008 at the University of Texas MD Anderson Cancer Center was evaluated. Clinicopathologic features of the patients are listed in Table E1 in the online supplement. The inclusion criteria were NSCLC histology, stage I to III, no neoadjuvant or adjuvant chemotherapy or radiotherapy, and absence of cancer within the 5 years previous to lung cancer surgery.

\section{Cell Lines}

YES1 status of all cell lines is listed in Table E2.

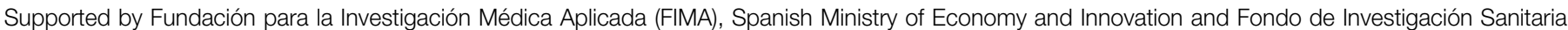

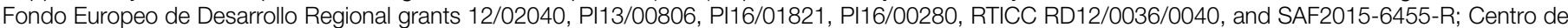

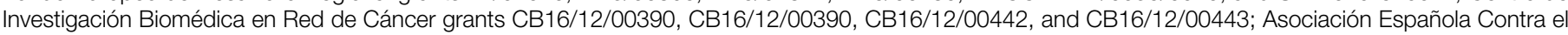

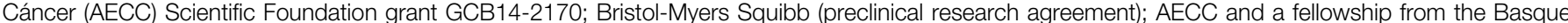

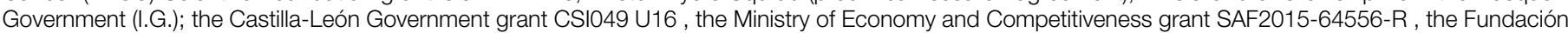

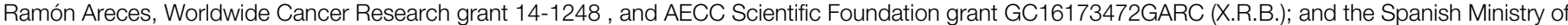
Science, Innovation and Universities (MCIU, RTI 2018-094507-B-100), La Caixa Foundation and Caja Navarra Foundation (F.L.).

Author Contributions: L.M.M. and J.A. conceived the experiments and supervised the work. I.G., M.J.P., C. Behrens, A.B.R., E.F., M.S.-C., and I.I.W.

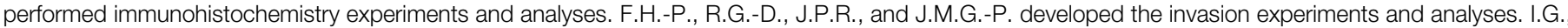

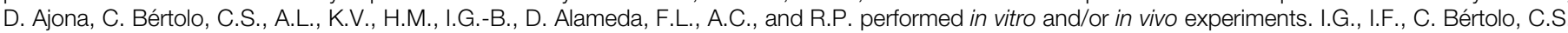

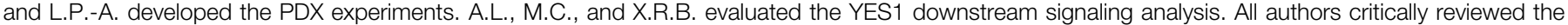
manuscript. 
A

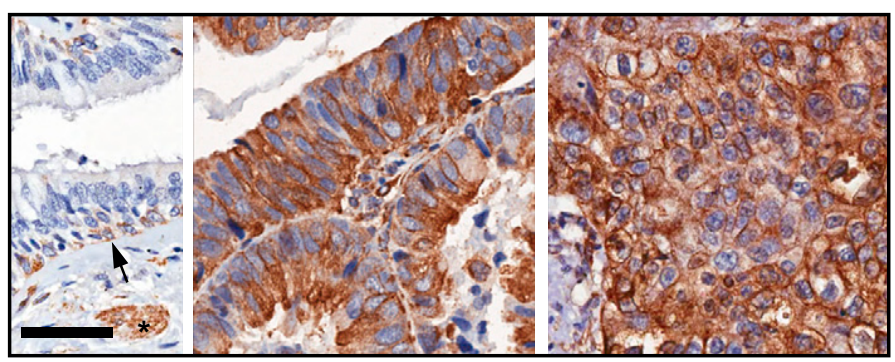

B

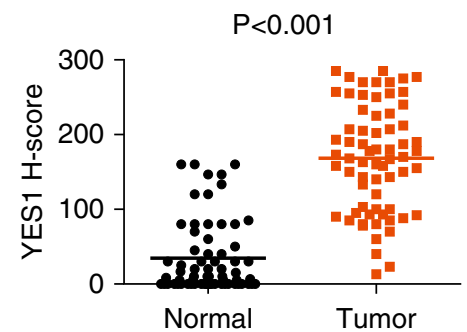

C

CIMA-CUN series

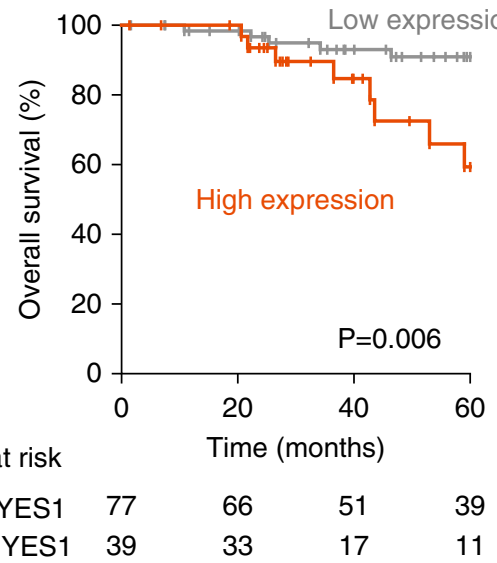

D

MDA Series

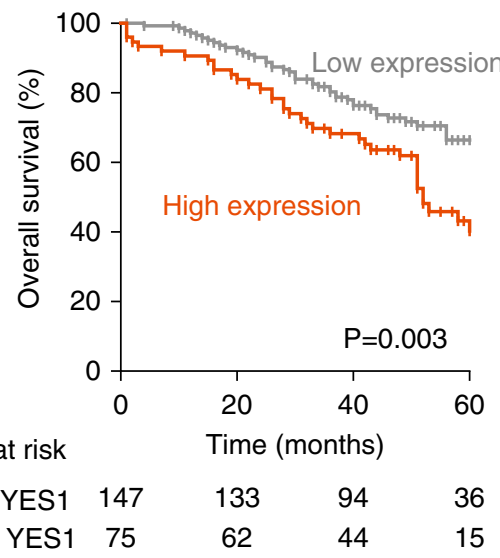

E

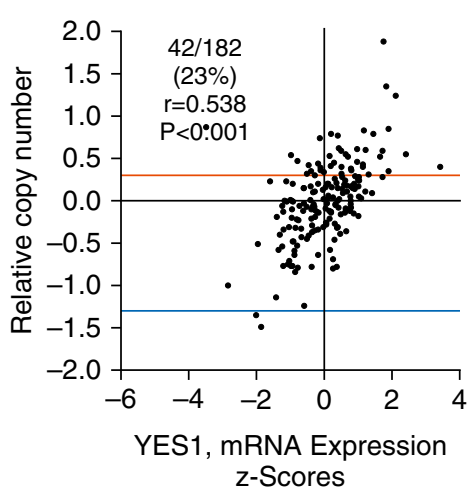

ADC TCGA

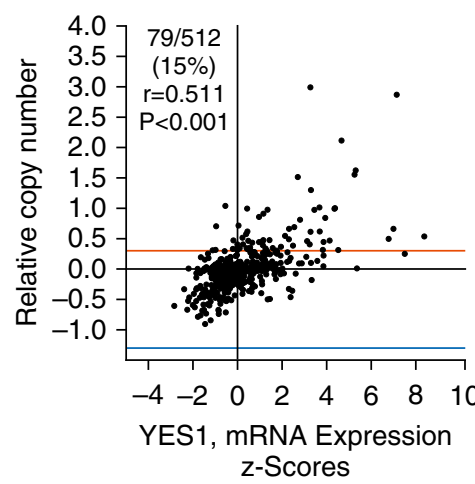

SCC TCGA

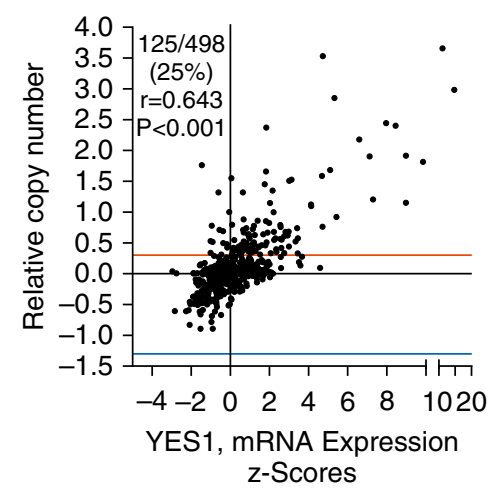

Figure 1. YES1 (v-YES-1 Yamaguchi sarcoma viral oncogene homolog 1) is associated with adverse prognosis in patients with non-small cell lung cancer (NSCLC). (A) Representative images of immunohistochemical analysis of YES1 immunostaining in bronchial epithelium (left panel), lung adenocarcinoma (ADC) (middle panel) and lung squamous cell carcinoma (SCC) (right panel). Basal cells (arrow) and smooth muscle cells (asterisk) showed YES1 immunoreactivity. High cytoplasmic (middle panel) and membrane (right panel) YES1 staining can be found in tumor cells. Scale bar, $50 \mu \mathrm{m}$. (B) YES1 expression was significantly higher in tumor cells than in paired nontumor tissue. ( $C$ and $D$ ) High YES1 expression is associated with adverse prognosis in NSCLC: Kaplan-Meier overall survival curves for YES1 expression and log-rank test in the Clínica Universidad de Navarra (CIMA-CUN) series (C) and MD Anderson Cancer Center series $(D)$. (E) In silico analysis based on Cancer Cell Line Encyclopedia (CCLE) and The Cancer Genome Atlas (TCGA) data demonstrated a high correlation between gene copy number (CN) and mRNA expression of YES1 in lung cancer cell lines and both patients with ADC and patients with SCC. Red lines define the limit of $\mathrm{CN}$ gains $\left(\log _{2} \mathrm{CN} / 2=0.3 ; \mathrm{CN}>2.5\right.$ copies), and blue lines define the limit of homozygous deletion ( $\log _{2} \mathrm{CN} / 2=-1.3 ; \mathrm{CN}<0.8$ copies). The Spearman coefficient $(r)$ was calculated to evaluate correlation between $\mathrm{CN}$ and $\mathrm{mRNA}$ expression. 
A

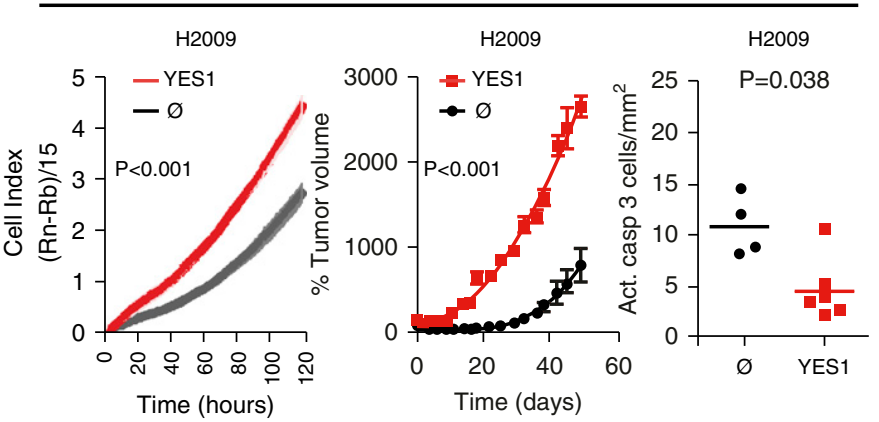

C

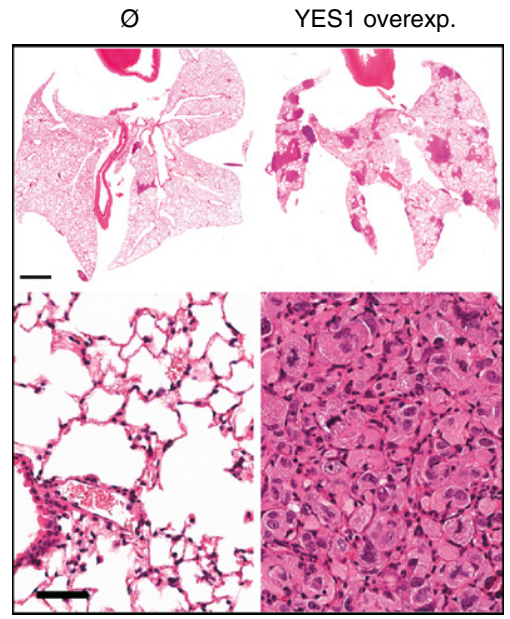

B

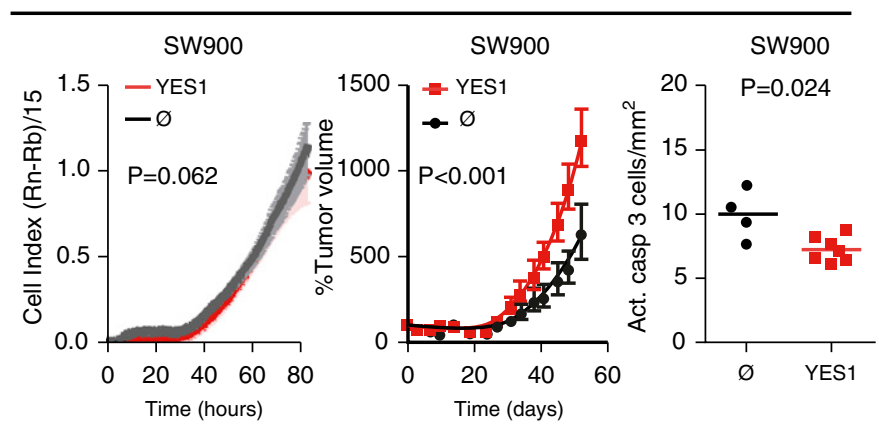

D

A549 (s.c.)

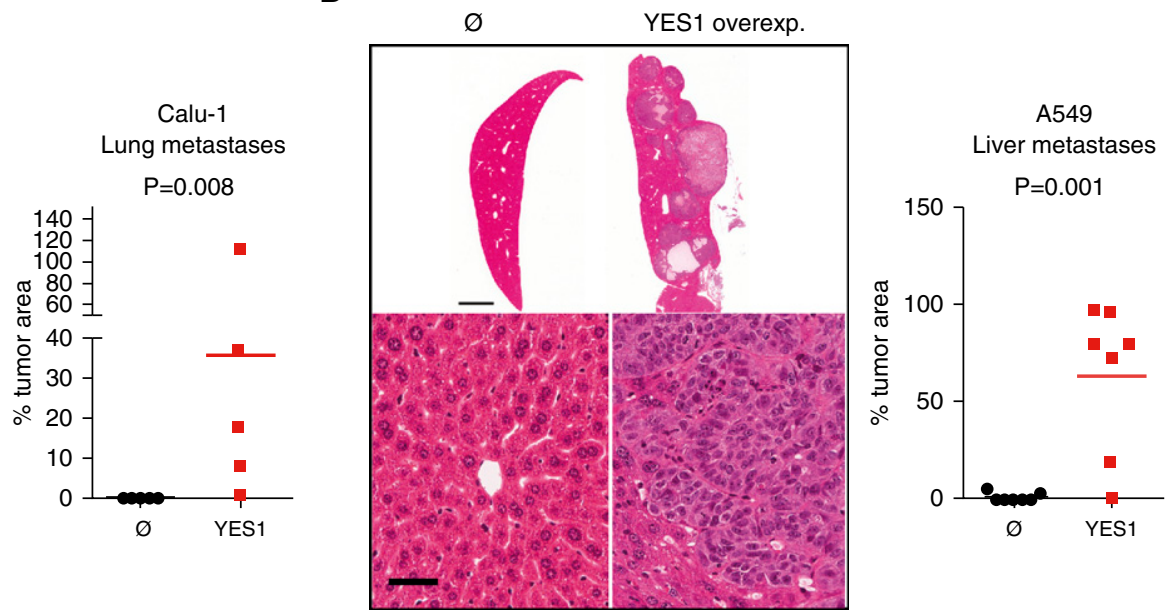

F A549 (i.c.)

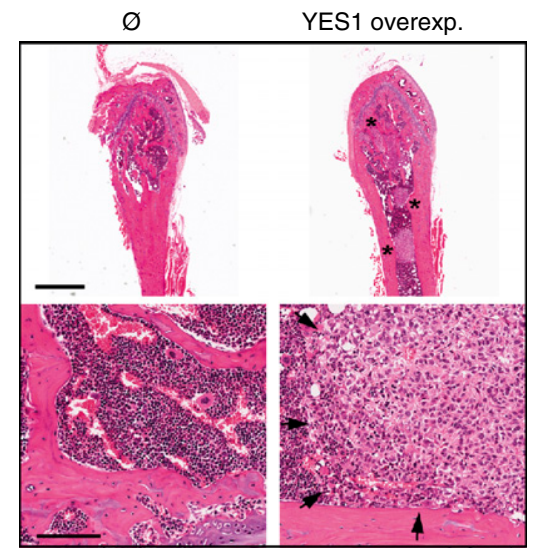

Figure 2. YES1 (v-YES-1 Yamaguchi sarcoma viral oncogene homolog 1) overexpression induces tumor growth and metastatic spreading. (A and $B$ ) Effect of constitutive YES1 overexpression in H2009 (A) and SW900 (B) cell lines. Left panels: YES1 overexpression induces in vitro cell proliferation only in HighYES1 cells as measured by XCELLigence real-time cell analyzer system. The experiments were done in triplicate and repeated three times. Middle panels: in vivo tumor growth of subcutaneously injected cells overexpressing YES1 was induced in both High YES1 and ${ }^{\text {Low }}$ YES1 cell lines. Data are presented as mean \pm SE ( $n \geqslant 4$ for all groups), and differences among groups were compared with the extra-sum-of-squares $F$ test. Right panels: YES1 overexpression reduced the number of apoptotic cells in subcutaneous tumors as measured by the number of positive activated (cleaved) caspase 3 cells per area. Empty vector transduced cells $(\varnothing)$ were used as controls. $n \geqslant 4$ for all groups. (C) Subcutaneous (s.c.) tumors of Calu- 1 cells overexpressing YES1 were able to metastasize to the lungs. Left panel, top: Representative histological images of metastatic growth in the lungs (scale bar, $2 \mathrm{~mm}$ ). Left panel, bottom: higher-magnification images (scale bar, $50 \mu \mathrm{m}$ ). Right panel: metastatic area in the lungs of mice subcutaneously injected with control or YES1-overexpressing Calu-1 cells ( $n=5$ for all groups). Tumor area was measured by using ImageJ analysis program. (D) YES1-overexpressing A549 


\section{Immunohistochemistry}

Two independent observers evaluated the intensity (1: weak staining; 2 : moderate staining; 3: strong staining) and extensiveness (the percentage of positive cells) of staining in all of the study samples. The evaluation of YES1 expression was performed using the $\mathrm{H}$-score system as previously described (23). Briefly, $\mathrm{H}$-score was calculated by adding the products of each intensity and the corresponding area. The range of possible scores was from 0 to 300 , and expression level was categorized as low and high using the upper tertile of the $\mathrm{H}$-score.

\section{Real-Time Quantitative PCR and Western Blotting}

Details are provided in the online supplement.

\section{YES1 Overexpression}

Details are provided in the online supplement.

\section{YES1 Stable Knockdown by CRISPR/Cas9 Editing}

Details are provided in the online supplement.

\section{SiRNAs}

Cells were transfected with two commercial siRNAs against YES1 (Invitrogen).

\section{MTT and Clonogenic Assays}

Details are provided in the online supplement.

\section{xCELLigence Proliferation Assay}

The xCELLigence real-time cell analyzer system (Roche Applied Science) was used to analyze cell proliferation. Each condition was performed in triplicate.

\section{Apoptosis Analyses}

Apoptosis was measured by annexin $\mathrm{V}$-propidium iodide double staining as previously described (24).

\section{Three-Dimensional Spheroid Invasion Assay}

The procedure was performed as previously described (25).

\section{In Vivo Mouse Models}

Cells were subcutaneously injected in $\mathrm{Rag} 2^{-/-} \mathrm{IL} 2 \mathrm{R} \gamma^{-/-}$mice and treated daily by oral gavage with $60 \mathrm{mg} / \mathrm{kg}$ dasatinib (kindly provided by Bristol-Myers Squibb) or vehicle ( $80 \mathrm{mM}$ citric acid, pH 2.1). PDX models were generated in Dr. Paz-Ares' laboratory and selected depending on histology and YES1 expression (Table E2). PDX tumors were treated with dasatinib or vehicle as previously described.

\section{Analysis of Metastatic Progression}

Metastatic potential of the A549-TM-YES1 (bioluminescence reporter vector) was evaluated by subcutaneous or intracardiac injection, as previously described (26).

\section{Statistical Analysis}

Correlation between YES1 CN and mRNA expression was evaluated by the Spearman rank test. Data obtained from cleaved-caspase 3 and Ki67 immunohistochemistry, analyses of metastases, and MTT (3-[4,5dimethylthiazol-2-yl]-2,5-diphenyltetrazolium bromide) and clonogenic assays were analyzed by the Mann-Whitney $U$ test. Tumor growth and cell proliferation data were fitted and compared using the extrasum-of-squares $F$ test (27). Kaplan-Meier survival curves and the log-rank test were used to examine differences in overall survival (OS) (defined as the date of first surgery to the last follow-up visit or to disease-related death). Follow-up period was restricted to 60 months in all cohorts. Multivariate analysis was performed using the Cox proportional hazards model. Statistical analysis was performed using Prism 5 software (GraphPad Inc.) and SPSS 22.0 (IBM).

\section{Results}

\section{YES1 is Amplified in Lung Cancer and Its Expression Correlates with Poor Clinical Outcome in Patients with NSCLC}

We first evaluated the expression of YES1 by immunohistochemistry in a cohort of 116 patients with NSCLC from CIMA-CUN. Low or negative YES1 staining was found in the cytoplasm of normal lung bronchiolar cells, with slightly higher levels in basal cells of the epithelium (Figure 1A, left panel). In tumor cells, cytoplasmic YES1 staining was found in all tumors (Figure 1A, middle and right panels), although membrane expression was also detected in some cases (Figure 1A, right panel). YES1 expression was significantly higher in tumor cells than in paired nonmalignant tissue (Figure 1B; $P<0.001)$

To determine whether YES1 expression predicted lung cancer-related survival, univariate and multivariate analyses were performed in the CIMA-CUN series. Patients with high YES1 protein expression showed significantly shorter OS $(P=0.006$; Figure 1C). Multivariate Cox regression analysis revealed that high YES1 protein expression was an independent predictor of shorter OS (hazard ratio, 0.198; confidence interval, 0.063-0.629; $P=0.006$ ) (Table E3). We validated the association between YES1 protein expression and OS in an independent cohort of 222 patients from the MD Anderson Cancer Center (Figure 1D). A strong association between high YES1 protein expression and poor survival was found $(P=0.003)$. More importantly, the multivariate analysis validated high YES1 expression as an independent poor prognostic factor in patients with NSCLC (hazard ratio, 0.541; confidence interval, 0.337-0.869; $P=0.011$ ) (Table E3).

We next evaluated the frequency of YES1 alterations in NSCLC cell lines and patients using public data from the Cancer Cell Line Encyclopedia project (28) and The Cancer Genome Atlas study (TCGA) (29) (Figure 1E). In the Cancer Cell Line Encyclopedia dataset, 23\% of cell lines showed YES1 amplification. Moreover, in the TCGA dataset of patients with NSCLC, YES1 gene amplification was found in 15\% of ADC tumors and $25 \%$ of SCC. Interestingly, we found a significant positive correlation between YES1 gene CN

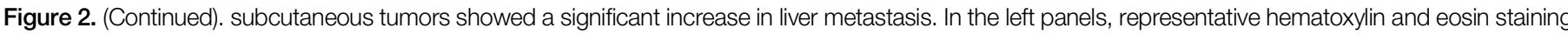

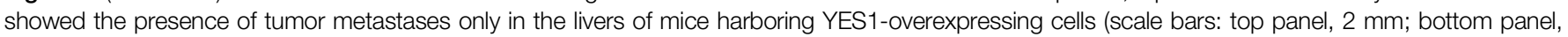

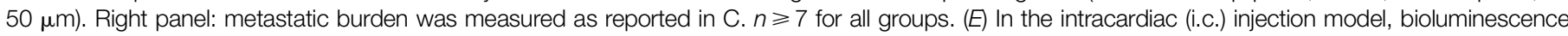

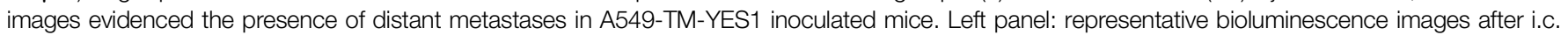

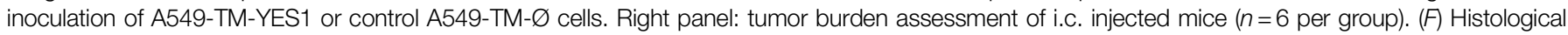

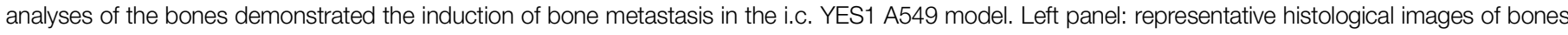

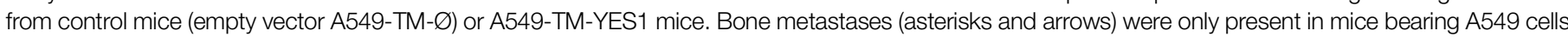

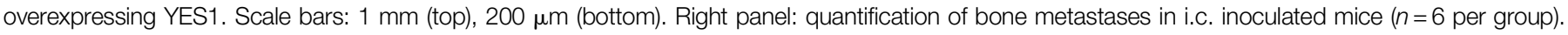



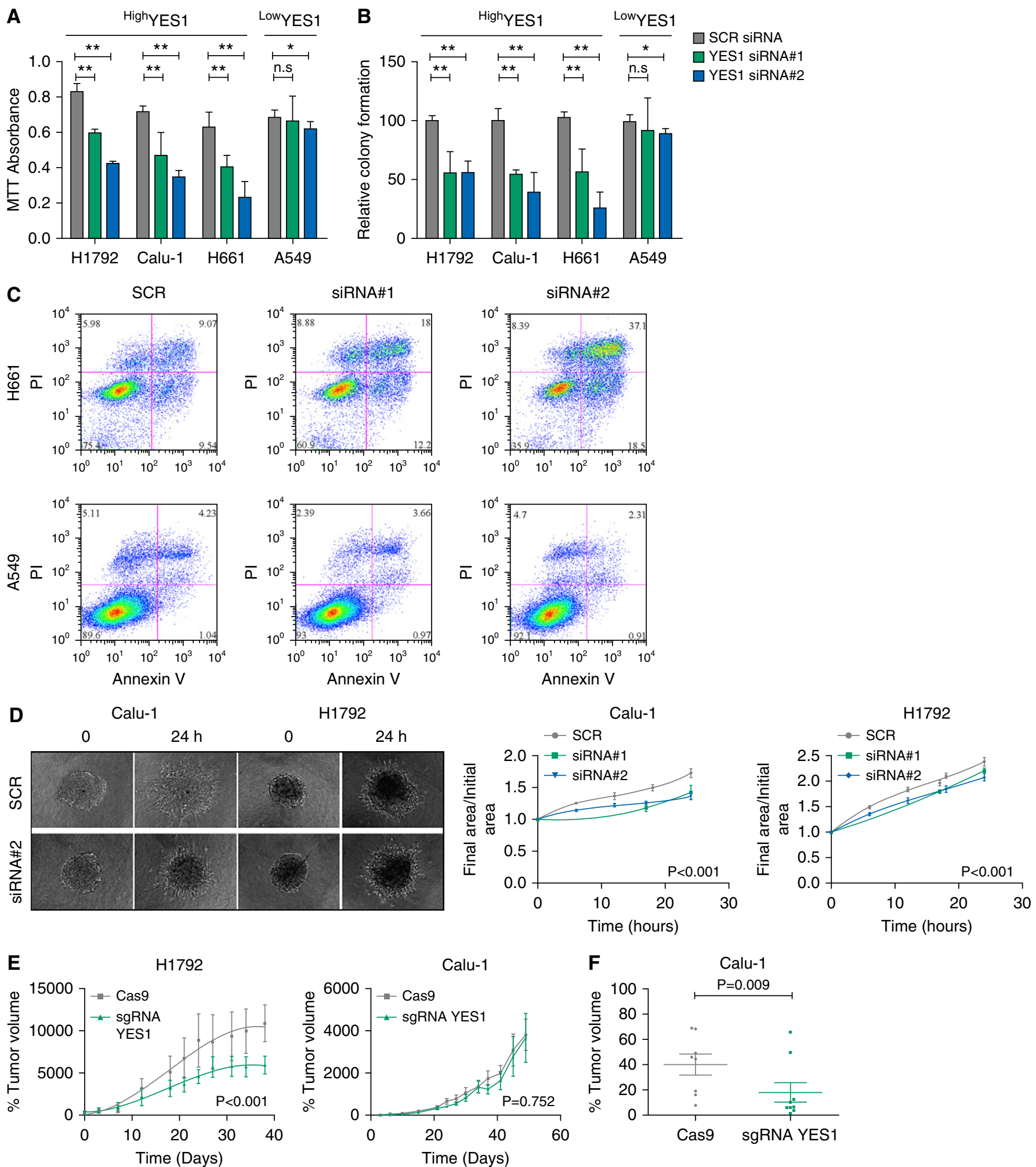

Figure 3. YES1 (v-YES-1 Yamaguchi sarcoma viral oncogene homolog 1) knockdown selectively regulates cell proliferation, apoptosis, and invasion in non-small-cell lung cancer (NSCLC) cell lines. ( $A$ and $B$ ) Knockdown of YES1 expression by two specific siRNAs selectively impairs cell proliferation ( $A$ ) and colony formation $(B)$ in ${ }^{H i g h}$ YES1 NSCLC cell lines. Data are presented as median \pm interquartile range from at least three independent experiments. ${ }^{\star} P<0.05$ and ${ }^{* \star} P<0.001$. (C) Representative images of flow cytometry analysis showing annexin $\vee$ and propidium iodide (PI) staining. In the ${ }^{\text {High }} Y E S 1$ cell line $\mathrm{H} 661$, siRNA treatment induced an increase in the percentage of early (annexin $\mathrm{V}^{+} / \mathrm{PI}^{-}$) and late apoptotic or necrotic cells (annexin $\mathrm{V}^{+} / \mathrm{PI}^{+}$). Conversely, no differences were found in the Low YES1 A549 cell line treated with control siRNA (SCR) or YES1-specific siRNAs (\#1 and \#2). (D) YES1 
and mRNA expression in lung cancer cell lines $(r=0.538 ; P<0.001)$ and in patients, in both ADC $(r=0.511 ; P<0.001)$ and SCC $(r=0.643 ; P<0.001)$. To analyze whether YES1 amplification co-occurred with the most frequent genomic alterations found in NSCLC, an in silico analysis of data from cBioPortal (30) was performed. No significant co-occurrences were found between YES1 amplification and other studied alterations in genes, including $A L K$, ROS1 (ROS proto-oncogene 1), BRAF (serine/threonine-protein kinase B-raf), KRAS (v-Ki-ras2 Kirsten rat sarcoma viral oncogene homolog), FGFR1 (fibroblast growth factor receptor 1), and TP53 (tumor protein $\mathrm{p} 53$ ), with the exception of DDR2 (discoidin domain receptor tyrosine kinase 2) amplification, which was found in 4 out of 2,621 patients with $\operatorname{ADC}(P=0.002)$, and EGFR $(P=0.009)$ and PIK3CA (phosphatidylinositol-4,5-bisphosphate 3-kinase catalytic subunit alpha) $(P=0.008)$ amplifications in 8 and 26 out of 1,176 patients with SCC, respectively.

These results demonstrate the prognostic value of YES1 protein expression in patients with NSCLC and indicate that genetic alterations of YES1 can be found in a highly significant subset of patients with NSCLC.

\section{YES1 Overexpression Significantly Induces Tumor Growth and Metastatic Spreading in In Vitro and In Vivo NSCLC Models}

To evaluate the protumorigenic role of YES1, stable YES1 overexpression was induced in lung cancer cell lines of different histological subtypes (ADC: H1792, H2009, A549, and SCC: Calu-1, SW900) (Figure E1). Constitutive YES1 overexpression significantly enhanced proliferation in cells harboring basal YES1 amplification and high protein expression $\left({ }^{\mathrm{High}}\right.$ YES1 cell lines, Table E2) (Figure 2A, left panel; Figure E2A). In marked contrast, no effect on cell proliferation was observed in cells without basal YES1 amplification ( ${ }^{\mathrm{Low}}$ YES1 cell lines, Table E2) (Figure 2B, left panel; Figure E2A).

We next evaluated the effect of YES1 overexpression in vivo. Subcutaneous models showed larger tumors from cells constitutively overexpressing YES1 as compared with control cells in all but one cell line (Calu-1, which showed an inverse correlation), independently of the basal status of YES1 (Figures 2A and 2B, middle panels; Figure E2B). Although no differences were observed in tumor proliferation (Figure E2C), YES1-overexpressing tumors showed a significant reduction in apoptosis, measured by cleaved-caspase 3 staining (Figures 2A and $2 \mathrm{~B}$, right panels; Figure E2D).

We further evaluated the presence of distant metastasis in NSCLC subcutaneous xenografts. In YES1-overexpressing Calu-1 cells, a clear induction of metastatic growth was found in the lungs (Figure $2 \mathrm{C} ; P=0.008$ ). In fact, no metastases were found in the control group. In the case of A549, both control and YES1-overexpressing cells metastasized to the lungs (Figure E3A). Notably, a significant induction of liver metastases in YES1-overexpressing cells was observed (Figure 2D; $P=0.001$ ). No metastatic growth was found in the mice injected with H2009, H1792, and SW900 cell lines. We next monitored metastatic burden by bioluminescence using A549 cells overexpressing YES1 and luciferase (A549TM-YES1). We confirmed that both control and YES1-overexpressing cells displayed lung metastases (Figure E3B). Furthermore, we verified the relevance of YES1 in metastatic spreading and found a significantly higher bioluminescence signal in the livers of A549-TM-YES1 mice than in control mice (Figure E3C; $P=0.041$ ). To further investigate the prometastatic activity of YES1 in lung cancer cells, we assessed an additional model of intracardiac injection and bone metastasis. Intracardiac inoculation of A549-TM-YES1 cells induced an increase in total tumor burden (Figure 2E; $P=0.030$ ).
Notably, bone metastases were only present in mice inoculated with YES1-overexpressing A549 cells (Figure 2F; $P=0.017$ ).

These findings show that YES1 facilitates the spreading of tumor cells and suggest that YES1 expression may have a relevant role in the regulation of the metastatic potential of NSCLC.

\section{YES1 Inhibition Selectively Impairs Proliferation, Survival, and Invasion of NSCLC Cells}

We then explored the therapeutic potential of YES1, hypothesizing that ${ }^{\text {High }}$ YES1 cells would be more sensitive to the depletion of the endogenous YES1 transcript. We first validated YES1 knockdown by two specific siRNAs (siRNA\#1 and \#2) (Figures E4A and E4B) and verified that SRC, FYN (FYN oncogene related to SRC), and LYN (v-YES-1 yamaguchi sarcoma viral-related oncogene homolog) expression was unaffected (Figures E4B and E4C). We found that YES1 inhibition significantly reduced cell proliferation and colony formation in ${ }^{\mathrm{High}}$ YES1 lung cancer cell lines, in clear contrast to the effect observed in ${ }^{\text {Low }}$ YES1 cells (Figures 3A and 3B). Accordingly, in ${ }^{\text {Low }}$ YES1 A549 and SW900 cell lines, apoptosis was not affected by YES1 downregulation, in contrast with ${ }^{\text {High }}$ YES1 H2009, H1792, and H661 cells (Figure 3C and Table E4). In ${ }^{\text {High }}$ YES1 Calu-1 and HCC95 cell lines, although apoptosis was not induced by YES1-specific siRNA transfection, this treatment seemed to be associated with increased autophagy levels, as measured by LC3 expression (Figure E5). We then measured the effect of YES1 downregulation on cell invasion by three-dimensional spheroid invasion assays, selecting those cell lines that were able to form regular spheres. YES1 knockdown inhibited the invasive potential in Calu-1 and H1792 cell lines ( ${ }^{\mathrm{High}}$ YES) (Figure 3D). We next sought to evaluate whether specific YES1 knockout by CRISPR

Figure 3. (Continued). downregulation inhibited three-dimensional (3D) invasion. Representative images from the 3D invasion assays of NSCLC cell spheroids embedded into a collagen matrix treated with control (SCR) or YES1-specific siRNAs (\#1 and \#2). The invasive area was determined by calculating the difference between the final area and the initial area $(t=0)$ using Image $J$ analysis program. All data were normalized to control-transfected cells. Data are presented as mean \pm SE from at least three independent experiments, and the differences were compared with the extra-sum-of-squares $F$ test. (E) YES1 abrogation by CRISPR (clustered regularly interspaced short palindromic repeats)/Cas9 (CRISPR-associated protein 9) editing reduced in vivo tumor growth of subcutaneously injected ${ }^{\text {High }}$ YES1 H1792 cells, whereas no effect was observed in High YES1 Calu-1 cell line. Data are presented as mean $\pm \mathrm{SE}(n \geqslant 5$ for all groups) and the extra-sum-of-squares $F$ test was used to compare tumor growth differences among groups. $(F)$ Metastatic area in the lungs of mice subcutaneously injected with Calu- 1 cells ( $n \geqslant 8$ for all groups). Tumor area was measured by using ImageJ analysis program. Metastatic growth in the lungs of Calu-1-injected mice was significantly inhibited by YES1 depletion as compared with control cells (Cas9). MTT=3-(4,5dimethylthiazol-2-yl)-2,5-diphenyltetrazolium bromide; sgRNA = single-guide RNA. 
A

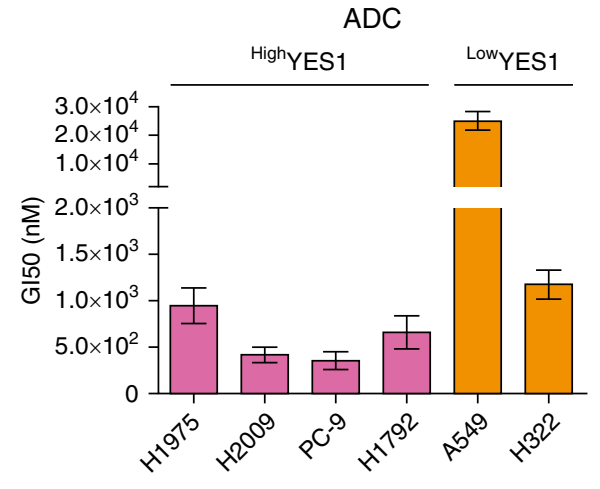

C

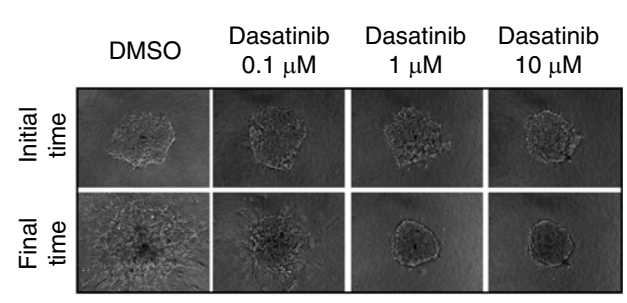

B

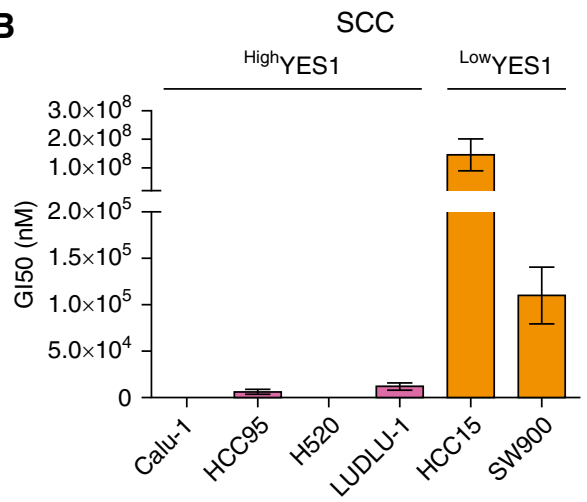

D

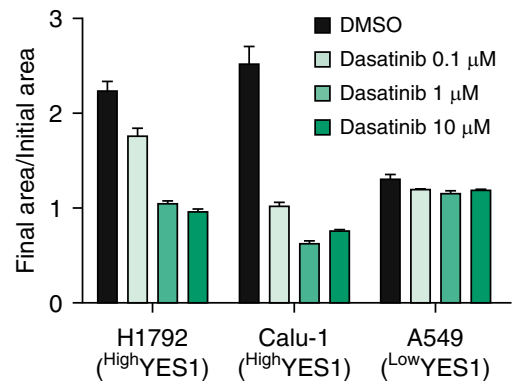

${ }^{\text {High YES1 }}$

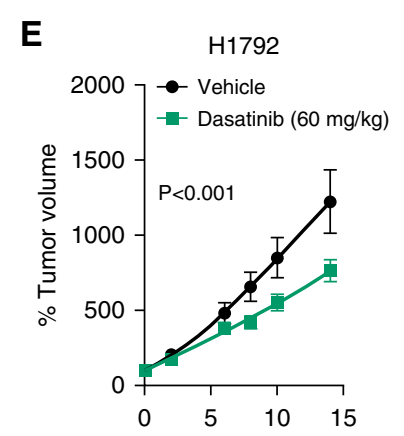

Days after treatment

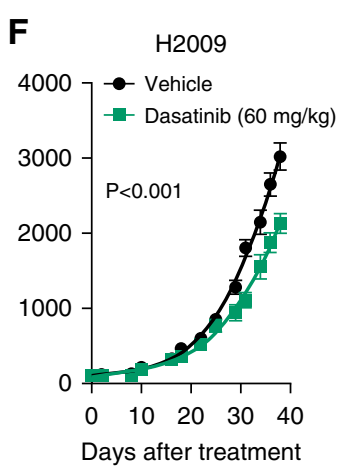

Days after treatment

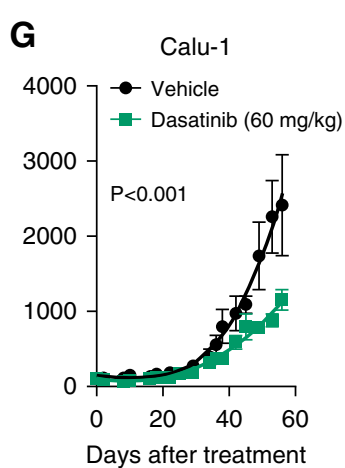

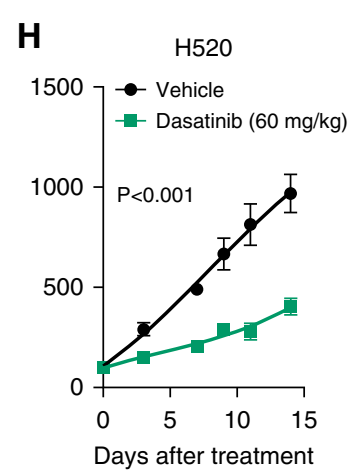

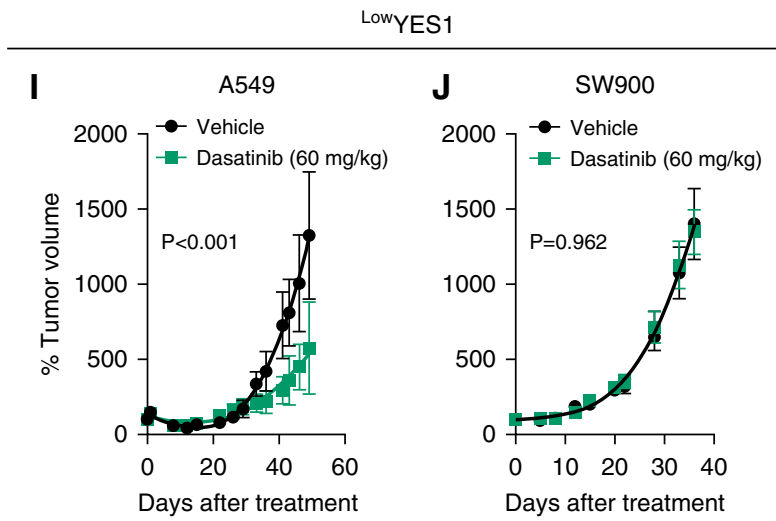

Figure 4. Dasatinib selectively and effectively blocks high YES1 (v-YES-1 Yamaguchi sarcoma viral oncogene homolog 1) cell line proliferation and invasion, as well as tumor growth in xenograft models. ( $A$ and $B$ ) Pharmacologic inhibition of YES1 by dasatinib significantly inhibited proliferation in ${ }^{H i g h}$ YES1 cell lines, whereas LowYES1 cells were more resistant to the treatment in both adenocarcinoma (ADC) (A) and squamous cell carcinoma (SCC) (B) subtypes. Cells were treated with increasing concentrations of dasatinib (10, 100, 1,000, 10,000, and 50,000 nM), and Gl50 was calculated based on cell proliferation curves obtained from MTT (3-[4,5-dimethylthiazol-2-yl]-2,5-diphenyltetrazolium bromide) assays (Figure E8). Data are presented as 
(clustered regularly interspaced short palindromic repeats)/Cas9 (CRISPRassociated protein 9) editing technology affects tumor growth in vivo. YES1 knockdown in ${ }^{\text {High }}$ YES1 H1792 and Calu-1 cell lines was confirmed by real-time quantitative PCR and Western blotting (Figure E6). YES1-depleted H1792 cell line significantly reduced tumor growth in subcutaneously injected mice (Figure 3E). In contrast, YES1 abrogation did not affect tumor growth of the Calu-1 cell line (Figure 3E). Interestingly, the histological examination of lungs and liver of these mice demonstrated a significant reduction of metastatic growth in the lungs of Calu-1-injected mice (Figure 3F); no metastases were found in the rest of analyzed samples. All these results strongly suggest that basal YES1 levels determine the antiproliferative and anti-invasive effects of YES1 downregulation.

To characterize the molecular mechanisms underlying the tumor-driving capabilities of YES1, we investigated the downstream signaling events triggered by YES1 (Figures E7A and E7B). We focused on the mTOR (mammalian target of rapamycin) pathway, as YES1 has been reported to be involved in AKT (protein kinase B [PKB]/Akt) phosphorylation (31). Although $\mathrm{p} 4 \mathrm{EBP} 1$ expression was not affected by YES1 expression, AKT phosphorylation (serine 473) and phosphorylation of the mTOR downstream effector S6K were downregulated in siRNAtreated cells (Figure E7A). Consequently, YES1 overexpression led to an increase in phospho-S6K (Figure E7B). Together, these data suggest that YES1 sustains mTOR pathway activity. The RAS/MAPK (Ras- or Mitogen-activated protein kinase) pathway was not altered by YES1 expression, because no ERK phosphorylation alterations were found.

\section{Pharmacological Inhibition of YES1 Impairs In Vitro and In Vivo Lung Cancer Growth}

To evaluate the feasibility of YES1 as a therapeutic target, we tested the effect of the SFK inhibitor dasatinib, a currently approved multitarget tyrosine kinase inhibitor that inhibits YES1 at nanomolar concentrations (32).

We first assessed the in vitro effect of dasatinib on cell proliferation in high and low YES1 cell lines (Figures 4A and 4B). Interestingly, in both ADC and SCC cell lines, dasatinib treatment inhibited proliferation in ${ }^{\mathrm{High}} \mathrm{YES} 1$ cell lines, whereas ${ }^{\text {Low }}$ YES1 cells were more resistant to dasatinib treatment (Figures $4 \mathrm{~A}$ and $4 \mathrm{~B}$; Figure E8). We also investigated the effect of YES1 pharmacological inhibition on in vitro cell invasion. Consistently, we found that dasatinib significantly blocked three-dimensional migration in ${ }^{\text {High }}$ YES1 cells (Calu-1 and H1792) in a dose-dependent manner (Figures $4 \mathrm{C}$ and 4D), whereas invasive capabilities of

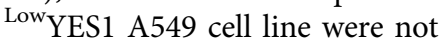
affected by dasatinib treatment (Figure 4D). These results suggest that YES1 status predicts the efficacy of dasatinib in vitro.

We next evaluated the pharmacological effectiveness of dasatinib in mice bearing subcutaneous tumors of a panel of ${ }^{\mathrm{High}}$ YES1 and ${ }^{\text {Low }}$ YES1 human lung cancer cells. In ${ }^{\text {High }}$ YES1 tumors, a significant tumor volume decrease was observed in dasatinibtreated mice when compared with vehicletreated control mice (Figures $4 \mathrm{E}-4 \mathrm{H}$ ). However, in ${ }^{\text {Low }}$ YES1 cell lines, differences in tumor growth were found only in one out of the two tested cell lines (Figures $4 \mathrm{I}-4 \mathrm{~J})$.

To assess whether YES1 amplification may be a predictor of response to SFK inhibitors, and to gain further insights into the potential therapeutic application of YES1 inhibition in patients carrying ${ }^{\text {High }}$ YES1 tumors, we used NSCLC PDX to explore the efficacy of dasatinib. Congruently, dasatinib significantly impaired tumor growth in ${ }^{\mathrm{High}} \mathrm{YES1}$ ADC or SCC PDX (Figures 5A-5C). Moreover, two out of three ${ }^{\text {Low }}$ YES1 PDX did not respond to dasatinib treatment (Figures 5D-5F), both in ADC and SCC models. In summary, these data suggest that YES1 status may be used as a stratification biomarker to select patients with NSCLC who may respond and benefit from SFK inhibitors.

\section{Discussion}

Although lung cancer is the archetype of genomics-driven oncology, a high proportion of patients cannot benefit from targeted therapies yet. This is especially relevant in patients with SCC who currently lack targeted molecular therapies. Our study provides evidence for the protumorigenic role of YES1 in NSCLC and reports the preclinical rationale for targeting this kinase, using its expression as an independent predictive marker of response to treatment. YES1 amplification has been recently reported as a new mechanism of resistance to EGFR inhibitors (17-19), suggesting that YES1-mediated signaling may exert an important role in lung cancer pathogenesis. However, the specific role of YES1 in lung cancer progression has not been explored yet. In this report, we show that YES1 alterations drive lung cancer progression and that high YES1 protein expression is associated with shorter overall survival in patients with NSCLC. We have also demonstrated that specific genetic and pharmacological YES1 inhibition reduces cell proliferation and invasion in vitro and tumor growth in vivo in ${ }^{\mathrm{High}} \mathrm{YES1}$ tumors.

A high proportion of patients with NSCLC harbor YES1 DNA CN alterations. In the TCGA series (29), 15\% of patients with ADC and $25 \%$ of patients with SCC show gains in the YES1 gene and a high mRNA expression. Interestingly, in a recent comprehensive analysis of NSCLC exome sequences and $\mathrm{CN}$ profiles, YES1 amplification was described as a recurrent alteration of lung SCC (8). In a previous study, we described a prognostic signature for lung ADC composed of seven-gene genomic alterations (4). Among the seven genes, YES1 and TYMS (thymidylate synthetase) presented the highest prognostic power. In the present study, we demonstrate that YES1 protein expression is an independent prognostic biomarker in lung cancer.

We have also demonstrated the driving role of YES1 in NSCLC carcinogenesis. Stable YES1 overexpression significantly induces cell proliferation in vivo and

Figure 4. (Continued). mean \pm SE from at least three independent experiments. ( $C$ and $D$ ) Dasatinib treatment blocked three-dimensional invasion of ${ }^{H i g h}$ YES1 Calu-1 $(C$ and $D)$ and H1792 $(D)$ cells in a dose-dependent manner. Invasion capability was not affected in Low YES1 A549 cell line by dasatinib

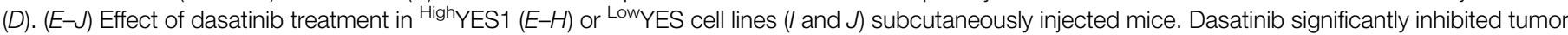
growth in High YES1 tumors, whereas no differences were found in the SW900 LowY YS1 tumors. Data are presented as mean \pm SE $(n \geqslant 6$ for all groups). The extra-sum-of-squares $F$ test was used to compare tumor growth differences among groups. 
${ }^{\text {High }}$ YES 1
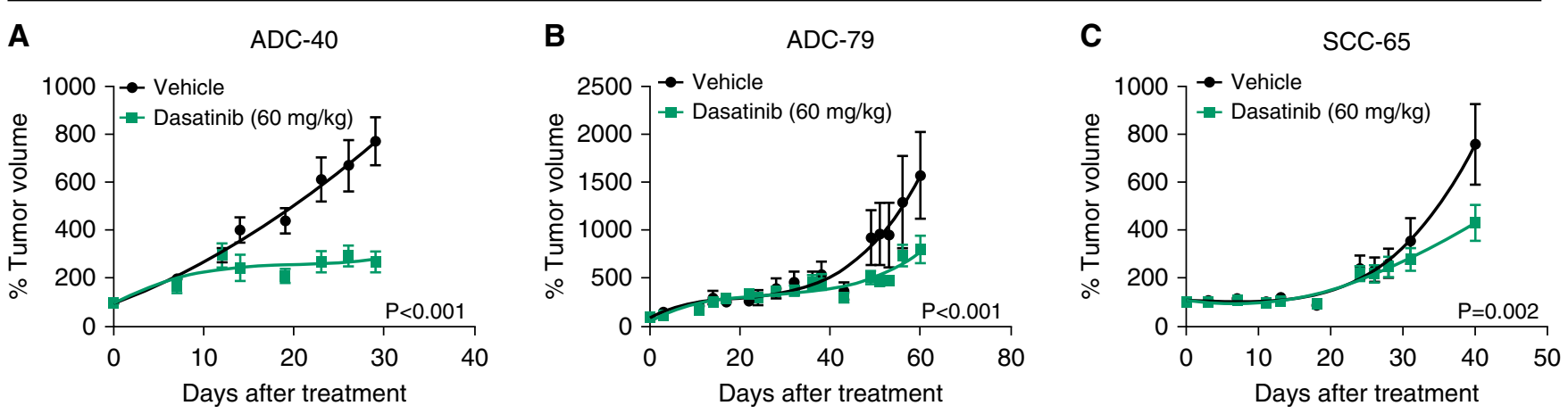

LoWYES 1
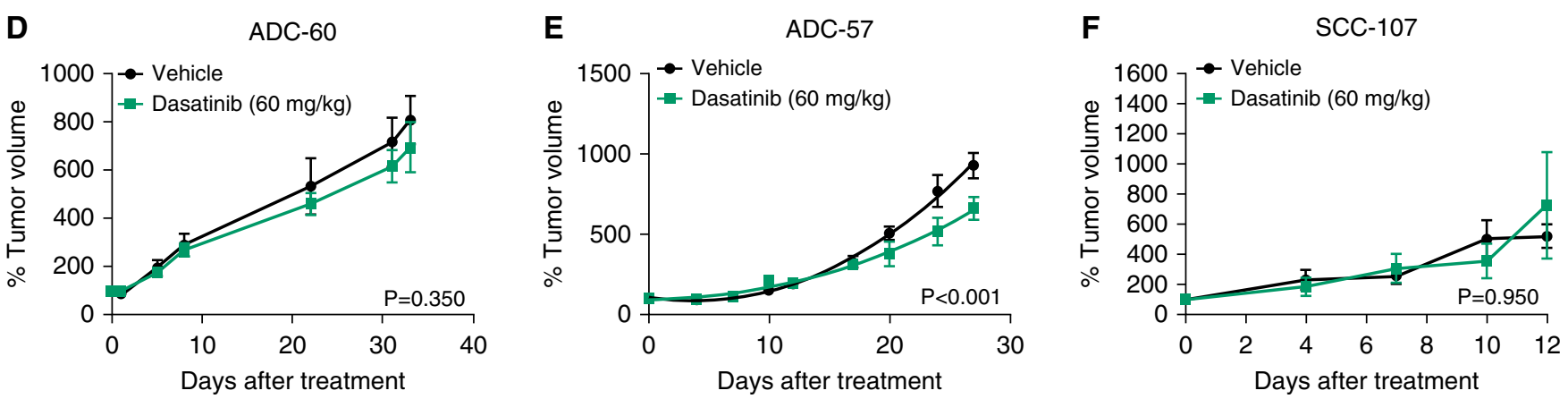

Figure 5. Dasatinib inhibits tumor growth in patient-derived xenograft (PDX) models depending on YES1 ( $\mathrm{v}$-YES-1 Yamaguchi sarcoma viral oncogene homolog 1) status. PDX models were developed and treated with dasatinib. Dasatinib treatment in High YES1 adenocarcinoma (ADC)-40, ADC-79, and squamous cell carcinoma (SCC)-65 PDX significantly inhibited tumor growth (A-C). In contrast, in Low YES1 ADC-60 and SCC-107 PDX models,

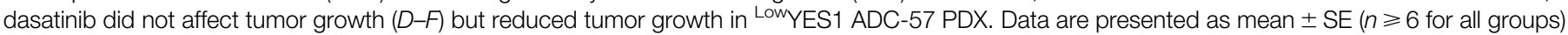
Tumor growth differences among groups were compared with the extra-sum-of-squares $F$ test.

in vitro, supporting the tumorigenic effect of this kinase. The increase on both proliferation and tumor growth is more prominent in NSCLC cell lines with basal high YES1 CN and protein expression. In fact, in the ${ }^{\text {Low }}$ YES1 A549 cell line, exogenous YES1 induction led to an increase in tumor growth in mouse models but not in vitro, suggesting that YES1 tumor expression may also regulate the microenvironment. In this regard, it has been reported in pancreatic tumors that the hyperactivation of the YES1 target FAK (focal adhesion kinase) induces a tumorprotective fibrotic and immunosuppressive microenvironment by inducing stromal expansion and tumor infiltration of immunosuppressive cells (33). It could be argued that, in ${ }^{\text {Low }}$ YES1 cell lines, the higher influence of YES1 induction in vivo may be due to its effect on the microenvironment more than on tumor cells per se. In addition, using two lung cancer mouse models, we have shown that YES1 promotes metastatic spread.
Subcutaneous tumors of NSCLC cells overexpressing YES1 developed metastasis to the lungs and liver. Moreover, intracardiac injection of cells harboring YES1 CN alterations led to increased tumor burden and induced metastases to the lung, liver, and bones. Conversely, YES1 abrogation by CRISPR/Cas9 gene editing was associated with reduced tumor burden and lower metastasis rate. In other tumor types, such as melanoma (34), colon carcinoma (35), and prostate cancer (36), YES1 and other SFK members have also been correlated with metastasis.

Furthermore, we have shown that YES1 knockdown selectively impairs cell growth and invasion and induces apoptosis in lung cancer cell lines harboring $\mathrm{CN}$ gains and high YES1 expression. In contrast, none of these effects were observed in cell lines with low expression and normal $\mathrm{CN}$ of this kinase, suggesting a genomic alteration-dependent effect.

Regarding molecular signaling, YES1 kinase has been previously reported to be implicated in FAK (36), YAP1 (37), and mTOR (31) pathways among others. In this work we found that the PI3K/AKT/mTOR pathway is an important mediator of YES1 signaling in NSCLC, as YES1 promotes S6K and AKT phosphorylation.

Given that our results determined that YES1 is a therapeutic target in high YES1expressing tumors, we evaluated the activity of the SFK inhibitor dasatinib in NSCLC models according to their YES1 status. Dasatinib is a multitarget tyrosine kinase inhibitor that inhibits SFKs at nanomolar concentrations, and it is also active against BCR-ABL (breakpoint cluster regionAbelson murine leukemia viral oncogene homolog 1), c-KIT (stem cell growth factor receptor Kit) and PDGFR $\beta$ (plateletderived growth factor receptor, beta polypeptide) (32). In lung cancer, unselected dasatinib trials were developed based on preclinical data on SRC inhibition (38). In this trial of 34 patients with advanced NSCLC, a striking response to first-line dasatinib therapy was found in 
one patient and long-lasting disease stabilization in four others, suggesting a potential subpopulation of patients sensitive to dasatinib. Unfortunately, no predictive markers were found, and a great effort has been made to identify biomarkers of response to dasatinib. Mutations in the $D D R 2$ kinase were proposed to predict dasatinib treatment in patients with SCC (39), but clinical trials failed to demonstrate this issue (40-42).

Our hypothesis is that dasatinib treatment may have therapeutic activity in those tumors harboring YES1 CN gains and high expression, functioning as a predictive biomarker for this therapy. This hypothesis is also supported by computational studies (43) suggesting that $\mathrm{CN}$ gains of a selected list of genes (which includes YES1) may serve as predictive markers for dasatinib treatment. However, the specific contribution of YES1 gene $\mathrm{CN}$ has so far never been investigated. In the present work, we report that the NSCLC (both ADC and SCC subtypes) sensitivity to dasatinib was closely associated with YES1 status. Indeed, cell proliferation and invasion were impaired by dasatinib in a dose-dependent manner mostly in ${ }^{H i g h}$ YES1 cells. Consistently, dasatinib also demonstrated to be an effective treatment for ${ }^{\text {High }}$ YES1 cells in our in vivo NSCLC models, being effective only in one out of two ${ }^{\text {Low } Y E S 1 ~ c e l l s, ~ A 549, ~ p r o b a b l y ~ b e c a u s e ~}$ of its high SRC expression. Expanding on these findings, we evaluated the efficacy of dasatinib in NSCLC PDX, confirming that this inhibitor led to robust tumor shrinkage in ${ }^{\mathrm{High}}$ YES1 tumors. Therefore, our results suggest that YES1 amplification may be considered a potentially useful predictive biomarker for dasatinib response. Basal high YES1 status is associated with dasatinib efficacy, although this association could not be excluded in low YES1 tumors, probably because of the expression of other SRC kinases in these tumors. We are currently exploring the potential interaction between YES1 overexpression and the immune landscape of the tumors as well as potential epistatic influences between YES1 amplification and other gene alterations. Importantly, YES1 amplification has been recently proposed as a new mechanism of resistance to EGFR inhibitors (17-19). Specifically, Fan and colleagues have demonstrated by transposon-based mutagenesis that acquired YES1 amplification is a mechanism of acquired resistance to EGFR TKIs (tyrosine-kinase inhibitors) afatinib, erlotinib, and osimertinib in lung ADC (19). Accordingly, patients with YES1 amplification may be selected for dasatinib treatment. The evaluation of YES1 status as a predictive marker of response to dasatinib in patients with NSCLC could also contribute to better selecting and stratifying patients. Ultimately, the refinement in the patient selection criteria may allow for clinical trials in which more adjusted working dosages of the SKF inhibitors would be tested to maximize treatment efficacy while minimizing toxicity. Furthermore, the development of specific YES1 inhibitors, rather than general SFK pan-inhibitors, may also increase the therapeutic efficacy in patients carrying ${ }^{\mathrm{High}}$ YES1 tumors. Finally, in clinical trials exploring the combination of dasatinib and immune checkpoint inhibitors such as the FRACTION (Fast Real-Time Assessment of Combination Therapies in ImmunoOncology)-lung study, YES1 expression may be used to assess efficacy of the combined treatment.

In summary, the results presented herein not only support the prognostic significance of YES1 but also, more importantly, demonstrate that YES1 is a driver of tumor growth and progression in a high percentage of patients with NSCLC. Thus, YES1 targeting emerges as a potential therapeutic strategy in patients with NSCLC harboring YES1 genetic alterations. Indeed, we have presented a comprehensive preclinical evaluation of the SFK inhibitor dasatinib, demonstrating its efficacy in ${ }^{\text {High }}$ YES1 tumors both in vitro and in vivo. Altogether, our studies provide support for the clinical evaluation of YES1 as a stratification biomarker to define a subset of patients who may potentially benefit from current or novel SFK inhibitors.

Author disclosures are available with the text of this article at www.atsjournals.org.

\begin{abstract}
Acknowledgment: The authors thank Carolina Zandueta for assistance with the mouse model of metastasis. They also thank Elizabeth Guruceaga and Víctor Segura from Bioinformatics Service, and the Morphology Department and Animal Production and Experimentation of the Center for Applied Medical Research for technical support.
\end{abstract}

\section{References}

1. Herbst RS, Morgensztern D, Boshoff C. The biology and management of non-small cell lung cancer. Nature 2018;553:446-454.

2. Thomas A, Liu SV, Subramaniam DS, Giaccone G. Refining the treatment of NSCLC according to histological and molecular subtypes. Nat Rev Clin Oncol 2015;12:511-526.

3. Politi K, Herbst RS. Lung cancer in the era of precision medicine. Clin Cancer Res 2015;21:2213-2220.

4. Aramburu A, Zudaire I, Pajares MJ, Agorreta J, Orta A, Lozano MD, et al. Combined clinical and genomic signatures for the prognosis of early stage non-small cell lung cancer based on gene copy number alterations. BMC Genomics 2015;16:752.

5. Kim LC, Song L, Haura EB. Src kinases as therapeutic targets for cancer. Nat Rev Clin Oncol 2009;6:587-595.

6. Rothschild SI, Gautschi O, Haura EB, Johnson FM. Src inhibitors in lung cancer: current status and future directions. Clin Lung Cancer 2010; $11: 238-242$

7. Rosti G, Castagnetti F, Gugliotta G, Baccarani M. Tyrosine kinase inhibitors in chronic myeloid leukaemia: which, when, for whom? Nat Rev Clin Oncol 2017;14:141-154.
8. Campbell JD, Alexandrov A, Kim J, Wala J, Berger AH, Pedamallu CS, et al.; Cancer Genome Atlas Research Network. Distinct patterns of somatic genome alterations in lung adenocarcinomas and squamous cell carcinomas. Nat Genet 2016;48:607-616.

9. Nakakuki K, Imoto I, Pimkhaokham A, Fukuda Y, Shimada Y, Imamura M, et al. Novel targets for the 18p11.3 amplification frequently observed in esophageal squamous cell carcinomas. Carcinogenesis 2002;23:19-24.

10. Brown J, Bothma H, Veale R, Willem P. Genomic imbalances in esophageal carcinoma cell lines involve Wnt pathway genes. World $\mathrm{J}$ Gastroenterol 2011;17:2909-2923.

11. Takeda T, Yamamoto H, Kanzaki H, Suzawa K, Yoshioka T, Tomida S, et al. Yes1 signaling mediates the resistance to trastuzumab/lap atinib in breast cancer. PLoS One 2017;12:e0171356.

12. lida M, Brand TM, Campbell DA, Li C, Wheeler DL. Yes and Lyn play a role in nuclear translocation of the epidermal growth factor receptor. Oncogene 2013;32:759-767.

13. Summy JM, Gallick GE. Src family kinases in tumor progression and metastasis. Cancer Metastasis Rev 2003;22:337-358.

14. Lee JH, Pyon J-K, Kim DW, Lee SH, Nam HS, Kim CH, et al. Elevated $\mathrm{c}-\mathrm{Src}$ and $\mathrm{c}-$ Yes expression in malignant skin cancers. J Exp Clin Cancer Res 2010;29:116. 
15. Ohkawa Y, Momota H, Kato A, Hashimoto N, Tsuda Y, Kotani N, et al. Ganglioside GD3 enhances invasiveness of gliomas by forming a complex with platelet-derived growth factor receptor $\alpha$ and yes kinase. J Biol Chem 2015;290:16043-16058.

16. Sato A, Sekine M, Virgona N, Ota M, Yano T. Yes is a central mediator of cell growth in malignant mesothelioma cells. Oncol Rep 2012;28: 1889-1893.

17. Ichihara E, Westover D, Meador CB, Yan Y, Bauer JA, Lu P, et al. SFK/FAK signaling attenuates osimertinib efficacy in both drugsensitive and drug-resistant models of EGFR-mutant lung cancer. Cancer Res 2017;77:2990-3000.

18. Yu HA, Suzawa K, Jordan E, Zehir A, Ni A, Kim R, et al. Concurrent alterations in EGFR-mutant lung cancers associated with resistance to EGFR kinase inhibitors and characterization of MTOR as a mediator of resistance. Clin Cancer Res 2018;24:3108-3118.

19. Fan P-D, Narzisi G, Jayaprakash A, Venturini E, Robine N, Smibert P, et al. YES1 amplification is a mechanism of acquired resistance to EGFR inhibitors identified by transposon mutagenesis and clinical genomics. Proc Natl Acad Sci USA 2018;115: E6030-E6038.

20. Agorreta J, Garmendia I, Pajares M, Ajona D, Alameda D, Behrens C, et al. YES1 kinase is a new therapeutic target in non-small cell lung cancer [abstract]. $J$ Thorac Oncol 2017;12:S446-S447.

21. Garmendia I, Agorreta J, Pajares MJ, Ajona D, Alameda D, Behrens C, et al. Dasatinib for the treatment of patients with non-small cell lung cancer harboring YES1 amplification [abstract]. Cancer Res 2017;77: LB-117.

22. Garmendia I, Bértolo C, Ferrer I, Pajares MJ, Ajona D, Paz-Ares L, et al. Dasatinib reduces tumor growth in xenograft models derived from human lung tumors with YES1 overexpression [abstract]. Cancer Res 2018;78:LB-084.

23. Pajares MJ, Agorreta J, Larrayoz M, Vesin A, Ezponda T, Zudaire I, et al. Expression of tumor-derived vascular endothelial growth factor and its receptors is associated with outcome in early squamous cell carcinoma of the lung. J Clin Oncol 2012;30:1129-1136.

24. Agorreta J, Hu J, Liu D, Delia D, Turley H, Ferguson DJ, et al. TRAP1 regulates proliferation, mitochondrial function, and has prognostic significance in NSCLC. Mol Cancer Res 2014;12:660-669.

25. Álvarez-Teijeiro S, Menéndez ST, Villaronga MÁ, Rodrigo JP, Manterola $\mathrm{L}$, de Villalaín L, et al. Dysregulation of mir-196b in head and neck cancers leads to pleiotropic effects in the tumor cells and surrounding stromal fibroblasts. Sci Rep 2017;7:17785.

26. Ajona D, Zandueta C, Corrales L, Moreno H, Pajares MJ, Ortiz-Espinosa $\mathrm{S}$, et al. Blockade of the complement $\mathrm{C} 5 \mathrm{a} / \mathrm{C} 5 \mathrm{aR} 1$ axis impairs lung cancer bone metastasis by CXCL16-mediated effects. Am J Respir Crit Care Med 2018;197:1164-1176.

27. Berraondo $P$, Nouzé $C$, Préville $X$, Ladant D, Leclerc $C$. Eradication of large tumors in mice by a tritherapy targeting the innate, adaptive, and regulatory components of the immune system. Cancer Res 2007;67:8847-8855.

28. Barretina J, Caponigro G, Stransky N, Venkatesan K, Margolin AA, Kim S, et al. The cancer cell line encyclopedia enables predictive modelling of anticancer drug sensitivity. Nature 2012;483:603-607.
29. Weinstein JN, Collisson EA, Mills GB, Shaw KR, Ozenberger BA Ellrott K, et al.; Cancer Genome Atlas Research Network. The cancer genome Atlas pan-cancer analysis project. Nat Genet 2013;45: 1113-1120.

30. Cerami E, Gao J, Dogrusoz U, Gross BE, Sumer SO, Aksoy BA, et al. The cBio cancer genomics portal: an open platform for exploring multidimensional cancer genomics data. Cancer Discov 2012;2: 401-404.

31. Merhi A, Delrée $P$, Marini AM. The metabolic waste ammonium regulates mTORC2 and mTORC1 signaling. Sci Rep 2017;7:44602.

32. Lombardo LJ, Lee FY, Chen P, Norris D, Barrish JC, Behnia K, et al. Discovery of N-(2-chloro-6-methyl- phenyl)-2-(6-(4-(2-hydroxyethyl)piperazin-1-yl)-2-methylpyrimidin-4- ylamino)thiazole-5-carboxamide (BMS-354825), a dual Src/Abl kinase inhibitor with potent antitumor activity in preclinical assays. J Med Chem 2004;47: 6658-6661.

33. Jiang $\mathrm{H}$, Hegde S, Knolhoff BL, Zhu Y, Herndon JM, Meyer MA, et al. Targeting focal adhesion kinase renders pancreatic cancers responsive to checkpoint immunotherapy. Nat Med 2016;22:851-860.

34. Liu W, Monahan KB, Pfefferle AD, Shimamura T, Sorrentino J, Chan KT, et al. LKB1/STK11 inactivation leads to expansion of a prometastatic tumor subpopulation in melanoma. Cancer Cell 2012;21:751-764.

35. Sancier F, Dumont A, Sirvent A, Paquay de Plater L, Edmonds T, David $G$, et al. Specific oncogenic activity of the Src-family tyrosine kinase c-Yes in colon carcinoma cells. PLoS One 2011;6:e17237.

36. Chatterii T, Varkaris AS, Parikh NU, Song JH, Cheng C-J, Schweppe $\mathrm{RE}$, et al. Yes-mediated phosphorylation of focal adhesion kinase at tyrosine 861 increases metastatic potential of prostate cancer cells. Oncotarget 2015;6:10175-10194.

37. Sourbier C, Liao P-J, Ricketts CJ, Wei D, Yang Y, Baranes SM, et al. Targeting loss of the Hippo signaling pathway in NF2-deficient papillary kidney cancers. Oncotarget 2018;9:10723-10733.

38. Johnson FM, Bekele BN, Feng L, Wistuba I, Tang XM, Tran HT, et al. Phase II study of dasatinib in patients with advanced non-small-cell lung cancer. J Clin Oncol 2010;28:4609-4615.

39. Hammerman PS, Sos ML, Ramos AH, Xu C, Dutt A, Zhou W, et al. Mutations in the DDR2 kinase gene identify a novel therapeutic target in squamous cell lung cancer. Cancer Discov 2011;1:78-89.

40. Brunner AM, Costa DB, Heist RS, Garcia E, Lindeman NI, Sholl LM, et al. Treatment-related toxicities in a phase II trial of dasatinib in patients with squamous cell carcinoma of the lung. J Thorac Oncol 2013;8:1434-1437.

41. Pitini V, Arrigo C, Di Mirto C, Mondello P, Altavilla G. Response to dasatinib in a patient with SQCC of the lung harboring a discoidreceptor-2 and synchronous chronic myelogenous leukemia. Lung Cancer 2013;82:171-172.

42. Terai H, Tan L, Beauchamp EM, Hatcher JM, Liu Q, Meyerson M, et al. Characterization of DDR2 inhibitors for the treatment of $D D R 2$ mutated nonsmall cell lung cancer. ACS Chem Biol 2015;10: 2687-2696.

43. Sos ML, Michel K, Zander T, Weiss J, Frommolt P, Peifer M, et al. Predicting drug susceptibility of non-small cell lung cancers based on genetic lesions. J Clin Invest 2009;119:1727-1740. 